2015-10

Graphene electrode modified with

electrochemically reduced graphene

oxide for label-free DNA detection

$\mathrm{Li}, \mathrm{B}$

http://hdl.handle.net/10026.1/5552

10.1016/j.bios.2015.05.034

Biosensors and Bioelectronics

Elsevier BV

All content in PEARL is protected by copyright law. Author manuscripts are made available in accordance with publisher policies. Please cite only the published version using the details provided on the item record or document. In the absence of an open licence (e.g. Creative Commons), permissions for further reuse of content should be sought from the publisher or author. 


\title{
Graphene electrode modified with electrochemically reduced gra- phene oxide for label-free DNA detection
}

\author{
Bing Li ${ }^{\mathrm{a}, \mathrm{b}, *}$, Genhua Pan ${ }^{\mathrm{a}, \mathrm{b}}$, Neil D. Avent ${ }^{\mathrm{b}}$, Roy B. Lowry ${ }^{\mathrm{c}}$, Tracey E. Madgett ${ }^{\mathrm{b}}$, \\ Paul L. Waines ${ }^{\mathrm{b}}$ \\ ${ }^{a}$ Wolfson Nanomaterials \& Devices Laboratory, Faculty of Science and Engineering, University of Plymouth, Devon PL4 8AA, UK \\ ${ }^{\mathrm{b}}$ Centre for Research in Translational Biomedicine, Faculty of Science and Engineering, University of Plymouth, Devon PL4 8AA, UK \\ ' School of Geography, Earth and Environmental Sciences, Faculty of Science and Egineering, University of Plymouth, Devon PL4 8AA, UK
}

\section{A R T I C L E I N F O}

Article history:

Received 5 March 2015

Received in revised form

4 May 2015

Accepted 13 May 2015

Available online 14 May 2015

Keywords:

Grapheme

Electrochemically reduced graphene oxide

Electrochemical sensor

Label-free DNA detection

\begin{abstract}
A B S T R A C T
A novel printed graphene electrode modified with electrochemically reduced graphene oxide was developed for the detection of a specific oligonucleotide sequence. The graphene oxide was immobilized onto the surface of a graphene electrode via $\pi-\pi$ bonds and electrochemical reduction of graphene oxide was achieved by cyclic voltammetry. A much higher redox current was observed from the reduced graphene oxide-graphene double-layer electrode, a $42 \%$ and $36.7 \%$ increase, respectively, in comparison with that of a bare printed graphene or reduced graphene oxide electrode. The good electron transfer activity is attributed to a combination of the large number of electroactive sites in reduced graphene oxide and the high conductivity nature of graphene. The probe ssDNA was further immobilized onto the surface of the reduced graphene oxide-graphene double-layer electrode via $\pi-\pi$ bonds and then hybridized with its target cDNA. The change of peak current due to the hybridized dsDNA could be used for quantitative sensing of DNA concentration. It has been demonstrated that a linear range from $10^{-7} \mathrm{M}$ to $10^{-12} \mathrm{M}$ is achievable for the detection of human immunodeficiency virus 1 gene with a detection limit of $1.58 \times 10^{-13} \mathrm{M}$ as determined by three times standard deviation of zero DNA concentration.
\end{abstract}

(c) 2015 Elsevier B.V. All rights reserved.

\section{Introduction}

Due to its high conductivity, large surface to volume ratio and biocompatibility, graphene (Novoselov et al., 2004) has attracted a huge amount of interest for the electrochemical detection of specific DNA sequences (Dong et al., 2010b; Lim et al., 2010). The advantages of a graphene electrochemical electrode include wide potential windows (Niwa et al., 2006), electrochemical inertness in catalyst (Novoselov et al., 2012) and good electro-catalytic activity. However, one of the challenges for biosensor applications is that the biological probe molecules cannot directly bond to the surface of pristine graphene in most cases due to the absence of hydrophilic functionalities. In addition, only a very limited number of electroactive sites are available on the surface of pristine graphene, which limits the sensitivity of the biosensor (Davies et al., 2005). Graphene oxide (GO), graphene decorated with randomly distributed oxygen-containing functionalities on both sides of the plane, has also been widely reported as a biosensor material due to

\footnotetext{
* Corresponding author at: Centre for Research in Translational Biomedicine Faculty of Science and Engineering, University of Plymouth, Devon PL4 8AA, UK.

E-mail address: bing.li@plymouth.ac.uk (B. Li).
}

its high chemical and electrochemical activity (Bo et al., 2011; Davies et al., 2005; Dreyer et al., 2010; Liu et al., 2010). However, these oxidized areas on the GO plane break the long-range conjugated network and $\pi$-electron cloud, leading to a degradation of carrier mobility and conductivity (Zhao et al., 2010). Tremendous work has so far been carried out to develop different materials with an aim to achieve the merit of high conductivity and chemical/electrochemical activity on the same electrode (Dong et al., 2010a; Wu et al., 2013). Among these materials, reduced graphene oxide ( $\mathrm{rGO}$ ) is believed to be one of the best candidates due to its reasonably reduced number of functionalities (Gao et al., 2009), a large number of remaining electroactive sites (Gómez-Navarro et al., 2007) and the structural similarity with graphene. However, the conductivity of a thick rGO layer on a normal glassy carbon electrode can never be comparable to pristine graphene (Su et al. 2009) and deleterious chemicals are always used during rGO production (Kotov et al. 1996; Shin et al. 2009).

In this article, we report a novel rGO-graphene double-layer electrochemical biosensor for label-free detection of a DNA sequence. A GO layer was directly immobilized onto a screen-printed graphene electrode and reduced to rGO via cyclic voltammetry (CV) cycles. No deleterious chemicals, complicated immobilization for signal enhancement were employed. By using this novel rGO- 
graphene double-layer electrode, substantially elevated redox currents have been observed due to its high conductivity and high electroactivity. Probe ssDNA was then immobilized onto rGO for the detection of target cDNA. To our knowledge, this is the first report of the unique performance of dsDNA-rGO interaction on rGO-graphene double-layer electrode. We have also demonstrated a wide linear dynamic range and a low detection limit for labelfree detection of the human immunodeficiency virus 1 (HIV1) gene. Such a label-free detection of DNA with low cost, high sensitivity and wide linear dynamic range achieved via non-covalent immobilized probe ssDNA will be extremely attractive in genetic diagnosis and pathology (Avent et al., 2009; Houdebine, 2007) as target labelling (Batchelor-McAuley et al., 2009; He et al., 2010), signal enhancing (Cao, 2014; Wan et al., 2010) and probe immobilizing processes (Bo et al., 2011; Hu et al., 2011) are no longer required.

\section{Experimental}

\subsection{Reagents and apparatus}

GO aqueous solution, containing $>80 \%$ monolayer GO with a concentration of $0.5 \mathrm{mg} / \mathrm{ml}$, was prepared by the Modified Hummers' method (Kovtyukhova et al., 1999). Graphene modified working electrodes with a diameter of $3 \mathrm{~mm}$ were fabricated by a screen printing technique. Lyophilized oligonucleotides were synthesized and then purified with high-performance liquid chromatography (HPLC). The sequence of fluorescent dye labelled HIV1 gene is 5'-AGTCAGTGTGGAAAATCTCTAGC-carboxyfluorescein-3' (Carboxyfluorescein (FAM) is a fluorescein based dye), its complementary ssDNA sequence (known as cDNA) is 5'GCTAGAGATTTTCCACACTGACT-3' and the non-complementary sSDNA sequence is 5'- CGCCCTCTTCTTGTGGATG-3'. All other chemicals, such as potassium chloride $(\mathrm{KCl})$, potassium ferricyanide $\left(\mathrm{K}_{3}\left[\mathrm{Fe}(\mathrm{CN})_{6}\right]\right)$, phosphate-buffered saline tablet (PBS), etc. at biochemical grade were purchased from Sigma Aldrich (Dorset, UK).

Electrochemical analysis was carried out at room temperature with a Keithley 2602A multichannel source meter combined with a Cascade Microtech probe station MPS 150. Fluorescence images were obtained from a Nikon 80i Epi-fluorescence microscope with a $489 \mathrm{~nm}$ excitation laser. Raman spectra were obtained from a XPLORA HORIBA system equipped with a $532 \mathrm{~nm}$ laser and integrated with an OLYMPUS BX41 microscope. X-ray photoelectron spectroscopy analysis was carried out using the Kratos AXIS ULTRA with a mono-chromated $\mathrm{Al} \mathrm{k \alpha} \mathrm{X}$-ray source (1486.6 eV) operated at $10 \mathrm{~mA}$ emission current and $12 \mathrm{kV}$ anode potential $(120 \mathrm{~W})$. The ULTRA was used in fixed analyser transmission (FAT) mode, with pass energy of $80 \mathrm{eV}$ for wide scans and pass energy $20 \mathrm{eV}$ for high resolution scans.

\subsection{Fabrication of rGO-graphene double-layer electrode}

GO aqueous solution was diluted by adding distilled water (DI) water to the concentrations of $0.05 \mathrm{mg} / \mathrm{ml}, 0.1 \mathrm{mg} / \mathrm{ml}, 0.15 \mathrm{mg} / \mathrm{ml}$, $0.2 \mathrm{mg} / \mathrm{ml}, 0.25 \mathrm{mg} / \mathrm{ml}$ and $0.3 \mathrm{mg} / \mathrm{ml}$. $20 \mu \mathrm{l}$ of the diluted GO solution above was carefully dripped onto the surface of graphene modified electrodes and dried at room temperature for $1 \mathrm{~h}$ to promote strong bonds with the graphene underneath. $\mathrm{An} \mathrm{Ag} / \mathrm{AgCl}$ electrode and a platinum electrode were used as the reference and counter electrodes, respectively. The GO was then electrochemically reduced in the ferricyanide system $(10 \mathrm{mM}$ potassium ferricyanide with $1 \mathrm{M} \mathrm{KCl}$ ) by a $\mathrm{CV}$ cycle with an applied potential range of $0.5-1.5 \mathrm{~V}$ and a scan rate of $100 \mathrm{mV} / \mathrm{s}$ (theoretically, the reduction of GO began at $-0.6 \mathrm{~V}$ and reached a maximum at $-0.87 \mathrm{~V})$. The reduction can be achieved by only one cycle as the thickness of GO is thin enough and this is an electrochemically irreversible procedure at the potential above (Ramesha and Sampath 2009). The rGO-graphene double-layer electrode was rinsed with DI water three times and then dried at room temperature. By controlling the following key parameters: the quality of GO solution ( $>80 \%$ monolayer), the amount of drop-casted GO, the same area of the hydrophobic polymer insulated working electrode and the same numbers of $\mathrm{CV}$ reduction cycles, no significant differences in the symmetry and intensities of redox currents can be found between the resulting electrodes (Ramesha and Sampath, 2009). For the fabrication of an electrode modified with only rGO, the screen-printed graphene layer was removed in ultrasonic bath, then GO was drop-casted and reduced to rGO following the method above.

\subsection{Interaction of DNA-GO and DNA hybridization}

Lyophilized oligonucleotides were firstly dissolved in PBS buffer as stockers ( $\mathrm{pH} 7.0$, prepared with PBS tablet). For the fluorescence signal analysis: FAM-labelled probe ssDNA was further diluted to a concentration of $50 \mathrm{~nm}$ (He et al. 2010) in PBS buffer. A $30 \mu \mathrm{l} \mathrm{ssDNA}$ solution was chosen as a reference. $10 \mu \mathrm{l}$ of $300 \mathrm{~nm}$ target cDNA and $10 \mu \mathrm{l}$ blank PBS buffer were added into the mixtures of $10 \mu \mathrm{l}$ of $50 \mathrm{~nm}$ ssDNA and $10 \mu \mathrm{l}$ of $0.05 \mathrm{mg} / \mathrm{ml} \mathrm{GO}$ solution, respectively. To ensure the probe ssDNA could hybridize with its cDNA and come off from the surface of GO, cDNA was at least five times more concentrated than the probe ssDNA. The restoration of the fluorescence signal took up to $1 \mathrm{~h}$ to happen. Compared with the hybridization of ssDNA-cDNA, a much longer time is required is due to the competition existing between GOssDNA and ssDNA-cDNA. Then $20 \mu \mathrm{l}$ solution from each tube was dripped onto the freshly cleaned glass slides and dried at room temperature.

For the electrochemical analysis: the immobilization of probe ssDNA was achieved by adding $10 \mu$ probe ssDNA solution with a concentration of $10^{-6} \mathrm{M}$ (Bo et al. 2011) onto the surface of the rGO-graphene double-layer electrodes and keeping them at $35^{\circ} \mathrm{C}$ for $30 \mathrm{~min}$. After that, the probe ssDNA modified electrodes were rinsed with the nuclease free water for 15 times to remove those ssDNA which were weakly bonded onto the electrode surface or free-stood in electrolyte solution. DNA hybridization was conducted by dripping $10 \mu \mathrm{l}$ of the target cDNA solution (concentration varied from $10^{-12} \mathrm{M}$ to $10^{-7} \mathrm{M}$ ) onto the surface of ssDNArGO-graphene electrodes and culturing at room temperature for $1 \mathrm{~h}$. Then the surfaces of these electrodes were thoroughly rinsed with the nuclease free water before carrying out the electrochemical measurements.

\subsection{Electrochemical measurements}

Both CV and DPV (differential pulse voltammetry) measurements were carried out in $10 \mathrm{mM}$ ferricyanide aqueous solution ( $1 \mathrm{M} \mathrm{KCl}$ as the supporting electrolyte) at room temperature. Scan rates of CV measurements were varied from $30 \mathrm{mV} / \mathrm{s}$ to $200 \mathrm{mV} / \mathrm{s}$ in the characterization of the rGO-graphene double-layer electrode and the scan rate of $100 \mathrm{mV} / \mathrm{s}$ was chosen for the rest of this work. The scan potential ranged from $0.8 \mathrm{~V}$ to $-0.4 \mathrm{~V}$. The pulse period for the DPV measurement was $0.4 \mathrm{~s}$, pulse width was $0.12 \mathrm{~s}$, pulse amplitude was $50 \mathrm{mV}$, step potential was $10 \mathrm{mV}$ and quiet time was $2 \mathrm{~s}$. 


\section{Results and discussion}

\subsection{Optimization of GO concentration}

The first key requirement in developing a rGO-graphene double-layer electrode is to obtain a homogeneous GO layer on the surface of graphene electrode with a good electrochemical reversibility, which can be evaluated by $I_{\mathrm{pa}} / I_{\mathrm{pc}}$, the ratio of the anodic and cathodic peak currents (Gavalas et al., 2004; Silva et al., 2014). The stacking and bonding of GO-graphene here is achieved via the interaction of the $\pi$-electrons provided by $s p^{2}$ hybridized carbon in the lattice of GO and graphene. Fig. 1(a) shows the CV characterizations of the electrodes modified with GO solutions of different concentrations. It can be seen that the intensities of the redox currents decreased from $2.27 \times 10^{-4} \mathrm{~A}$ to $7.42 \times 10^{-5} \mathrm{~A}$ (a decrease of $67.3 \%$ ) with the increase of GO concentration from 0 to $0.3 \mathrm{mg} / \mathrm{ml}$, indicating that the surface of the graphene electrodes has been successfully modified. However, the GO layer is not "the thicker, the better" here. The analysis of $I_{\mathrm{pa}} / I_{\mathrm{pc}}$ showed the degradation of electrochemical reversibility and GO uniformity when the concentration of GO was higher than $0.15 \mathrm{mg} / \mathrm{ml}$, as shown by the average of three parallel independent trials in Fig. 1(b), where a drop of $I_{\mathrm{pa}} / I_{\mathrm{pc}}$ from $\sim 0.99$ to 0.9 occurred. This is also shown by the increasing peak separation as a function of GO concentrations. These results suggest that using GO with the concentration of $0.15 \mathrm{mg} / \mathrm{ml}$ is preferred in forming a homogeneous GO layer on the surface of graphene electrodes without losing its electrochemical reversibility in the ferricyanide system.

After the GO modification, the electroactive area of the electrode surfaces were estimated using the Randles-Sevcik Equation (Bard and Faulkner, 1980)

$I=\left(2.69 \times 10^{5}\right) A D^{1 / 2} n^{3 / 2} v^{1 / 2} C$

where, $I$ stands for the intensity of redox current, $A$ is electroactive area in $\mathrm{cm}^{2}, D$ stands for the diffusion coefficient of ferricyanide in $\mathrm{cm}^{2} \mathrm{~s}^{-1}, n$ is the number of electrons transferred in the redox reaction (in this case 1 ), $v$ is scan rate in $\mathrm{Vs}^{-1}$ and $C$ is the concentration of ferricyanide in $\mathrm{mol} \mathrm{L}^{-1}$. In this case, a decrease of $54 \%$ the electroactive area can be seen in Fig. 1(b) when the concentration of GO was $0.15 \mathrm{mg} / \mathrm{ml}$, indicating the increased area coverage/thickness of GO on the electrode surface. This is attributed to the insulating nature of GO due to its $s p^{2}$ hybridized graphene lattice surrounded by the randomly distributed defects (oxidized lattice with oxygen-containing functionalities) (Pandey et al., 2008), which can block the electron pathways between $s p^{2}$ carbon areas and in turn stop carrier transport (Zhao et al., 2010).

\subsection{Characterization of rGO-graphene double-layer electrode}

A comparison of Raman spectra obtained from electrodes modified with graphene, $\mathrm{GO}$ and rGO-graphene double-layer is shown in Fig. 2(a). After the immobilization of the GO layer onto a graphene electrode, the intensity ratio of the D and G bands in the Raman spectra $\left(I_{\mathrm{D}} / I_{\mathrm{G}}\right)$ increased from 0.02 (blue) to 0.8 (black). Also, the 2D band decreased significantly, indicating that the number of defective sites on the surface of electrode had significantly increased (Kim et al. 2014). The $I_{\mathrm{D}} / I_{\mathrm{G}}$ further increased to 1.28 (red) after the electrochemical reduction of GO, indicating that the GO layer on the surface of the graphene electrode had been effectively reduced. According to Lucchese et al. (Lucchese et al., 2010), $I_{\mathrm{D}} / I_{\mathrm{G}}$ is a function of the average distance between defective sites $\left(D_{\mathrm{f}}\right)$. It increases with an increase of $D_{\mathrm{f}}$ from 0 to $4 \mathrm{~nm}$, peaking at $4 \mathrm{~nm}$, and then decreases with the further increase of $D_{\mathrm{f}}$. As the oxygen-containing functionalities on the GO surface had been partly removed during the CV cycles, the size of the $s p^{2}$ hybridized domains (the distance between $D_{\mathrm{f}}$ ) within GO layers had consequently increased.

XPS has been used to quantitatively characterize the electrochemical reduction of GO. Fig. 2(b) shows the wide region XPS spectra of graphene electrodes modified with GO (black) and rGO (red) respectively. Both spectra contain $\mathrm{C}$, O signals with trace amounts of $\mathrm{K}, \mathrm{Cl}$ and $\mathrm{N}$ from the electrolyte. In comparison with the XPS of a GO modified graphene electrode, a significant intensity decrease for oxygen can be seen from that of a rGO modified graphene electrode. By extracting the corresponding sensitivities at $1486.6 \mathrm{eV}$, the atomic ratio of oxygen to carbon $(\mathrm{O} / \mathrm{C}$ ratio) decreased from $31 \%$ to $9 \%$. The $\mathrm{C} 1 \mathrm{~s}$ regional high-resolution spectra are also shown in the insets. The $\mathrm{C} 1 \mathrm{~s}$ for the GO-graphene electrode consists of three major peaks centred at $284.5 \mathrm{eV}, 287 \mathrm{eV}$ and $288.8 \mathrm{eV}$, corresponding to $s p^{2}$ and $s p^{3} \mathrm{C}-\mathrm{C}$ bonds in the graphene lattice, the $\mathrm{C}=\mathrm{O}$ bond in alkoxy and epoxy and the $\mathrm{C}=\mathrm{O}$ bond in carboxyl respectively (bottom inset, fitted by dash-dot line). After the electrochemical reduction of GO in ferricyanide, the peak intensities related to all the oxygen-containing functionalities decreased, especially that related to alkoxy and epoxy at $287 \mathrm{eV}$ (top inset, fitted by dash line). From these results, it can be confirmed that the GO has been effectively reduced during the CV cycles. Note that no structural damage to the graphene underneath has been introduced, in contrast to those violent or deleterious modification procedures reported by other groups (Teixeira et al., 2014), therefore, the unique electrical properties of graphene are

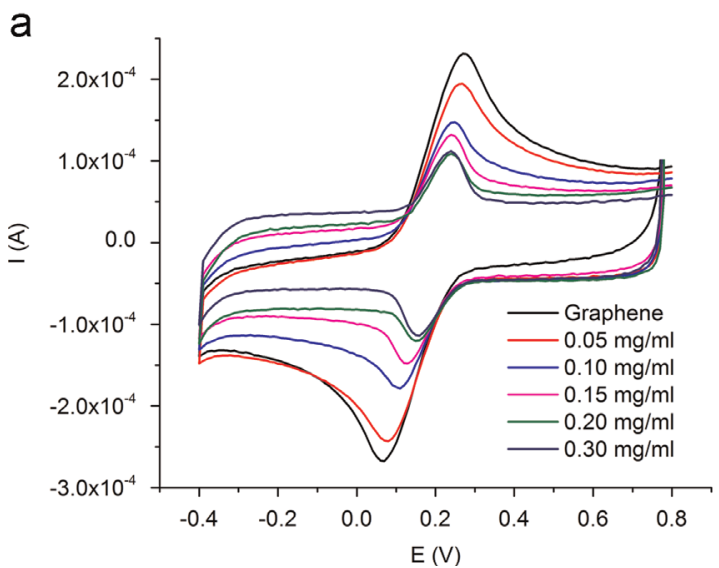

b

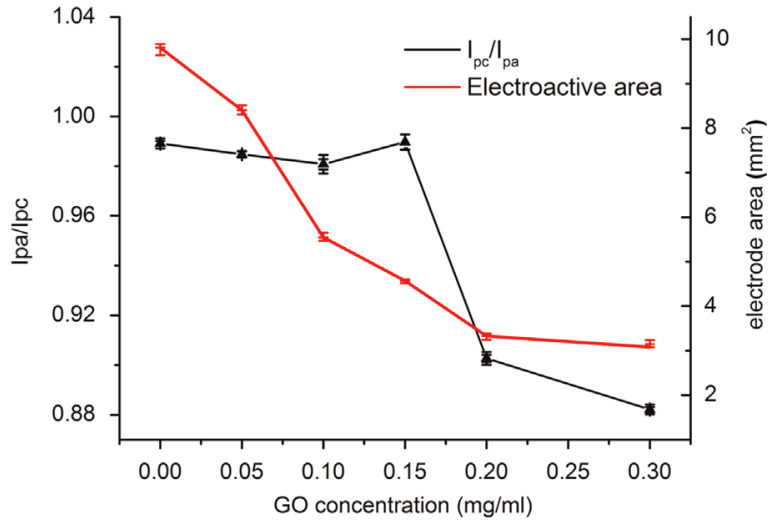

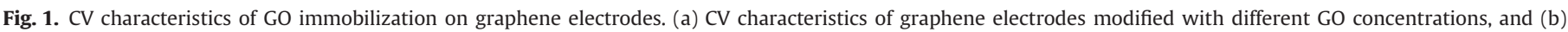

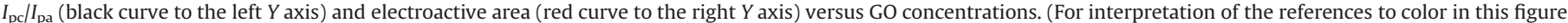
legend, the reader is referred to the web version of this article.) 
a

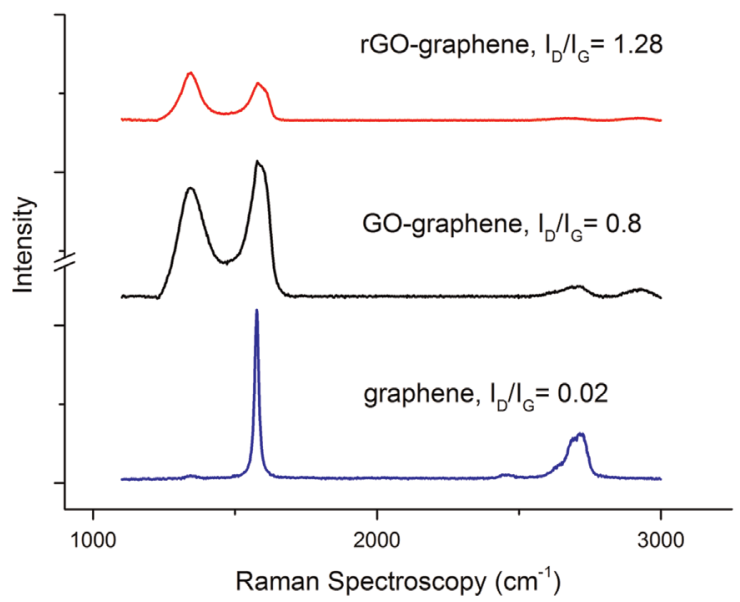

b

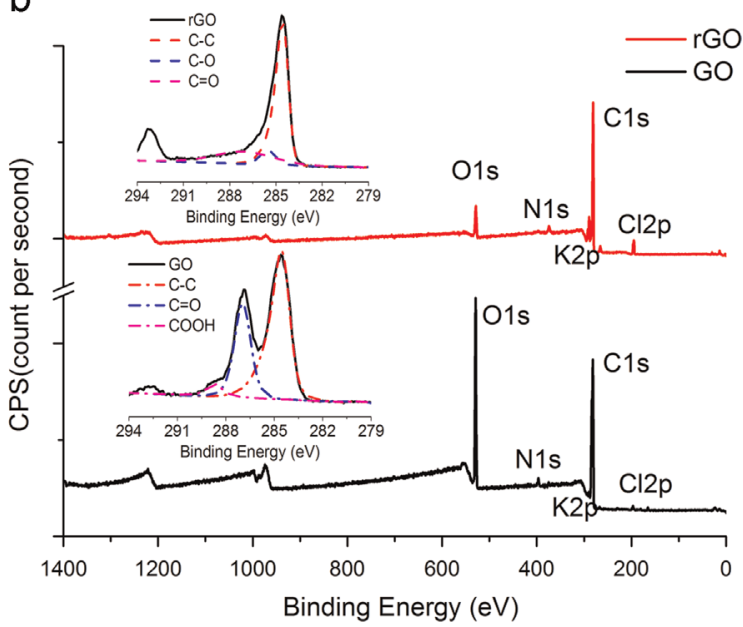

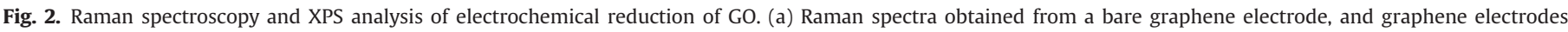

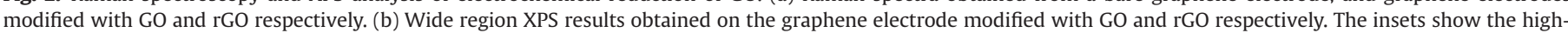
resolution spectra of $\mathrm{C} 1 \mathrm{~s}$ region accordingly. (For interpretation of the references to color in this figure, the reader is referred to the web version of this article.)

maintained. We therefore anticipate that the intensities of redox currents will be increased for the rGO-graphene double-layer electrode.

Fig. 3(a) shows a comparison of CV characteristics of four different layers-modified glassy carbon electrodes (graphene, rGO, GO-graphene and rGO-graphene). The superiority of using a rGOgraphene double-layer in electrode modification can be clearly seen from this set of results. The CV of the graphene electrode (black curve) shows an $I_{\mathrm{pa}}$ value of $2.204 \times 10^{-4} \mathrm{~A}$ and an $I_{\mathrm{pc}}$ value of $-2.247 \times 10^{-4} \mathrm{~A}$. After the modification with GO, the redox currents sharply decreased to $4.808 \times 10^{-5} \mathrm{~A}$ and $-4.809 \times 10^{-5} \mathrm{~A}$ (green curve). If the glassy carbon electrode is modified with only rGO, $I_{\mathrm{pa}}$ and $I_{\mathrm{pc}}$ are $2.297 \times 10^{-4} \mathrm{~A}$ and $-2.303 \times 10^{-4} \mathrm{~A}$ (blue curve). In contrast to all these, the rGOgraphene double-layer electrode shows much higher values of $I_{\mathrm{pa}}$ and $I_{\mathrm{pc}}$ of $3.138 \times 10^{-4} \mathrm{~A}$ and $-2.966 \times 10^{-4} \mathrm{~A}$ respectively (red curve).

The rGO layer contains a large number of defects inherited from the GO layer that are distributed within the rGO lattice and acting as the electroactive sites for donating or receiving electrons during the redox processes of ferricyanide (Du et al., 2010). Therefore, the rGO-graphene double-layer electrode not only shows a higher electron transport rate on the surface of the electrode compared

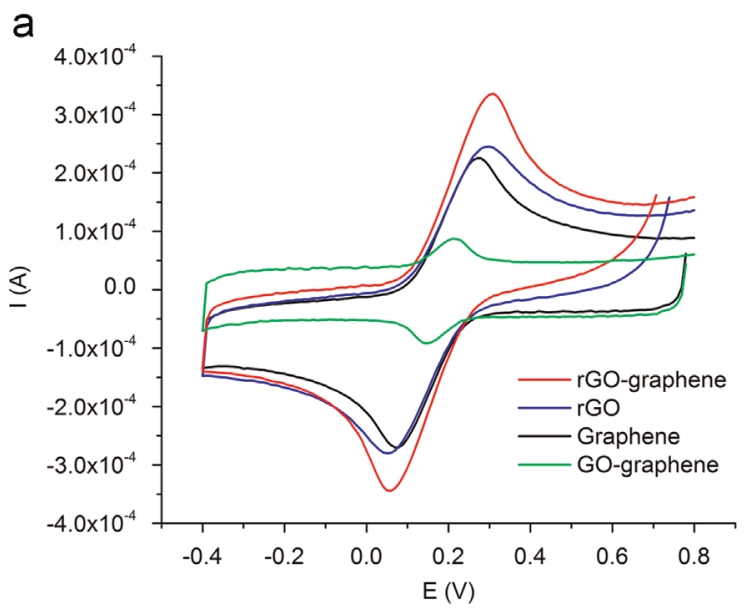

with those modified with rGO, pristine graphene or GO-graphene, but also provides a higher electron exchange rate at the electrode/ electrolyte interface for the ferricyanide system. By taking the combinational advantage of both the high conductivity of graphene and the high electroactivity of rGO, the intensity of the redox current obtained on the rGO-graphene double-layer electrode increased by $42 \%, 36 \%$ and $552 \%$ (calculated from corresponding curves in Fig. 3(a)) respectively, compared to those obtained on rGO, graphene and GO-graphene electrodes. Using the Randles-Sevcik Equation, the calculated electroactive areas for rGO, graphene, GO-graphene, and rGO-graphene electrodes were $9.8 \mathrm{~mm}^{2}, 9.7 \mathrm{~mm}^{2}, 2.0 \mathrm{~mm}^{2}$ and $12.8 \mathrm{~mm}^{2}$, respectively. Hence, the synergistic effect of using rGO-graphene in enhancing the electroactive area is significant.

Fig. 3(b) shows the effect of scan rate on CVs of a rGO-graphene double-layer electrode. These voltammagrams all show highly symmetric redox currents. The intensities of the redox currents show a linear correlation with the square root of scan rate (shown in inset of Fig. 3(b)), indicating a fast electrochemical process by the diffusion of ferricyanide and no definitive adsorption of ferricyanide on the surface of corresponding electrode, which is important for applications in biosensing.

b

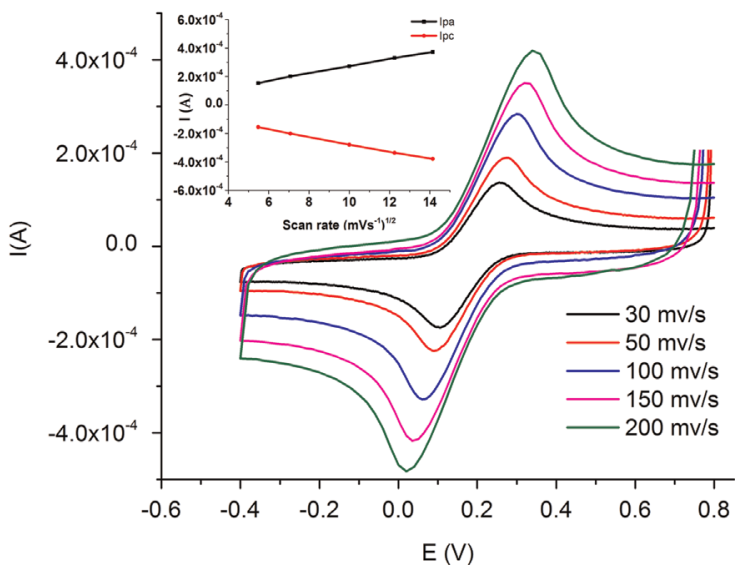

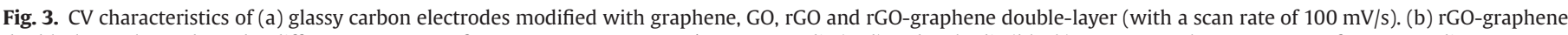

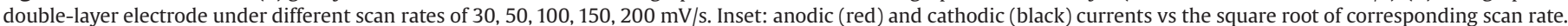
(For interpretation of the references to color in this figure legend, the reader is referred to the web version of this article.) 

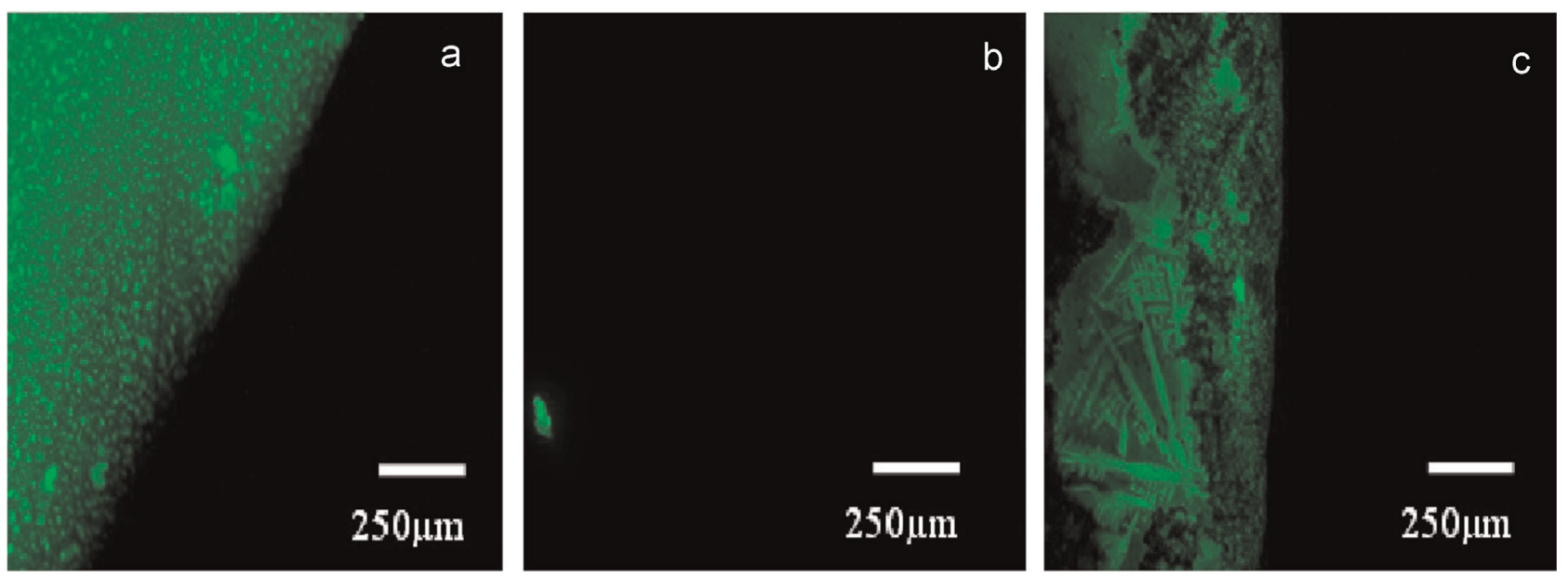

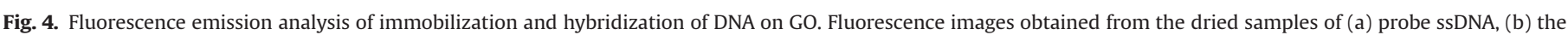
mixture of probe ssDNA and GO, and (c) the mixture of probe ssDNA, GO and target cDNA on glass slides. Laser excitation: 480 nm, signal emission: 520 nm.

\subsection{Fluorescence emission analysis of DNA-GO interaction}

The effectiveness of immobilizing ssDNA onto GO surface and hybridizing with its target cDNA is indirectly demonstrated by the following fluorescence emission analysis. Fig. 4 shows a comparison of the fluorescence emission images obtained on FAM-labelled probe ssDNA, the mixture of GO and FAM-labelled probe ssDNA and the mixture of GO and dsDNA (after probe ssDNA hybridized with its cDNA). $30 \mu \mathrm{l}$ of $50 \mathrm{~nm}$ FAM-labelled ssDNA dried on a glass slide shows strong fluorescence emission under a $480 \mathrm{~nm}$ excitation laser in the absence of GO, shown in Fig. 4(a). The fluorescence signal was nearly $100 \%$ quenched in the presence of $10 \mu \mathrm{l}$ of $0.15 \mathrm{mg} / \mathrm{ml} \mathrm{GO}$, shown in Fig.4(b) (the illuminant dot in this image is a particle used to ensure focusing on the sample surface), which implies that ssDNA has been effectively immobilized onto the GO surface (He et al., 2010). 30 min after adding $10 \mu \mathrm{l}$ of $300 \mathrm{~nm}$ target cDNA into the mixture of GO and probe ssDNA, the fluorescence emission became visible again, as shown in Fig. 4(c), which implies that the interaction between dsDNA and GO had been weakened.

The quenching of the fluorescence signal indicated that the interaction of SsDNA and GO, which was due to the $\pi-\pi$ ( $\pi$ electrons from graphene and those from DNA nucleobases) bonds, was very strong and able to bring ssDNA tightly onto the surface of GO. As a result, the emission energy, which supposed to be released as fluorescence signal, was therefore quenched due to the long-range nanoscale energy transfer property of graphene oxide. When the probe ssDNA hybridized with its cDNA and formed a duplex structure, nucleobases of FAM-labelled dsDNA are shielded by the negatively charged phosphate backbone (Chang et al., 2010; He et al., 2010; Lu et al., 2009). Therefore, the interaction between dsDNA and GO was significantly disturbed and weakened and in turn lead to the restoration of the fluorescence signal (Lu et al., 2009). The results imply that the electrochemical response will be consequently changed as the different negativity of ssDNA and dsDNA on electrode surface.

\subsection{Label-free detection of target $c D N A$}

The immobilization of probe ssDNA and the detection of its cDNA on rGO-graphene double-layer electrodes are qualitatively monitored by the changes in $\mathrm{CV}$ redox currents. Fig. 5 shows the CV characteristics of ssDNA-rGO-graphene (red) and dsDNA-rGOgraphene (blue) with reference to that of a plain rGO-graphene double-layer electrode (black). The plain electrode shows the large redox currents with an $I_{\mathrm{pa}}$ value of $2.55 \times 10^{-4} \mathrm{~A}$ and an $I_{\mathrm{pc}}$ value

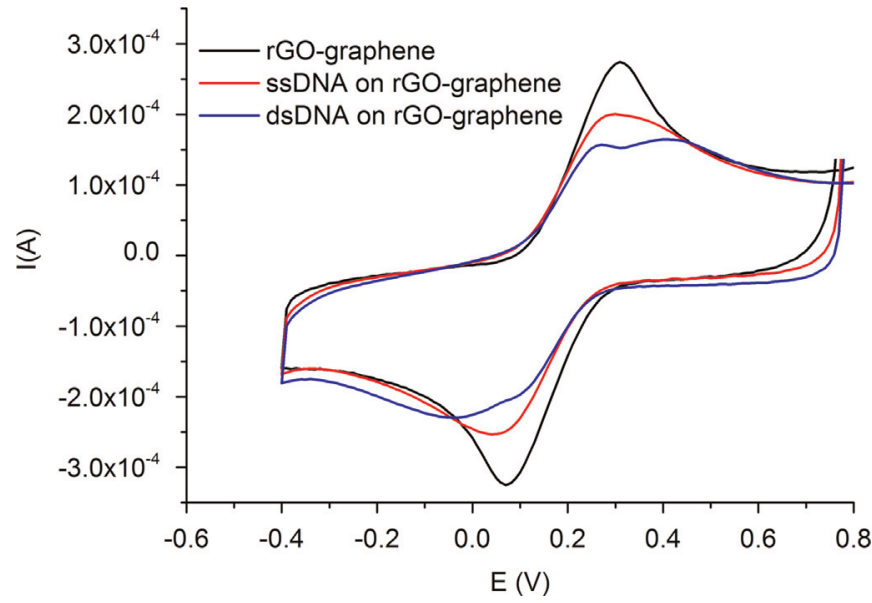

Fig. 5. CV characteristics of electrodes modified with rGO-graphene double-layer (black), ssDNA on rGO-graphene (red) and dsDNA on rGO-graphene (blue) (the concentration of target cDNA here is $100 \mathrm{nM}$ and the scan rate is $100 \mathrm{mV} / \mathrm{s}$ ). (For interpretation of the references to color in this figure legend, the reader is referred to the web version of this article.)

of $-2.69 \times 10^{-4} \mathrm{~A}$. After the probe ssDNA was bonded onto the surface of rGO, the redox currents decreased to $1.77 \times 10^{-4} \mathrm{~A}$ and $1.97 \times 10^{-4}$ A respectively. After ssDNA hybridized with its cDNA, these values further decreased to $1.31 \times 10^{-4} \mathrm{~A}$ and $-1.55 \times 10^{-4} \mathrm{~A}$. The other pair of redox peaks in Fig. 5 is attributed to the partially released dsDNA at a target concentration of $\geq 10^{-7} \mathrm{M}$ and these peaks became negligible when the concentration of target cDNA was $\leq 10^{-8} \mathrm{M}$. This phenomenon is attributed to the structural changes of bio-functional surface: ssDNA firstly bonded onto the surface of rGO via $\pi$ electrons provided by the purine/pyrimidine rings in DNA molecules and $s p^{2}$ hybridized carbon in graphene lattice, which leads to the negatively charged phosphate backbone facing away from the electrode surface (Patil et al., 2009). Therefore, an electrostatic repulsion effect occurred between the negatively charged phosphate backbones and the negatively charged electroactive-species (ferricyanide here), which in turn resulted in a lower electron transport rate (shown by the decrease in redox currents). With the addition of target cDNA onto the electrode surface, hybridization occurred and the interaction between dsDNA and the surface of rGO has been weakened (as explained in Fig. 4). However, we found that dsDNA did not completely come off from the electrode surface and had been "free standing" in the electrolyte solution as reported 


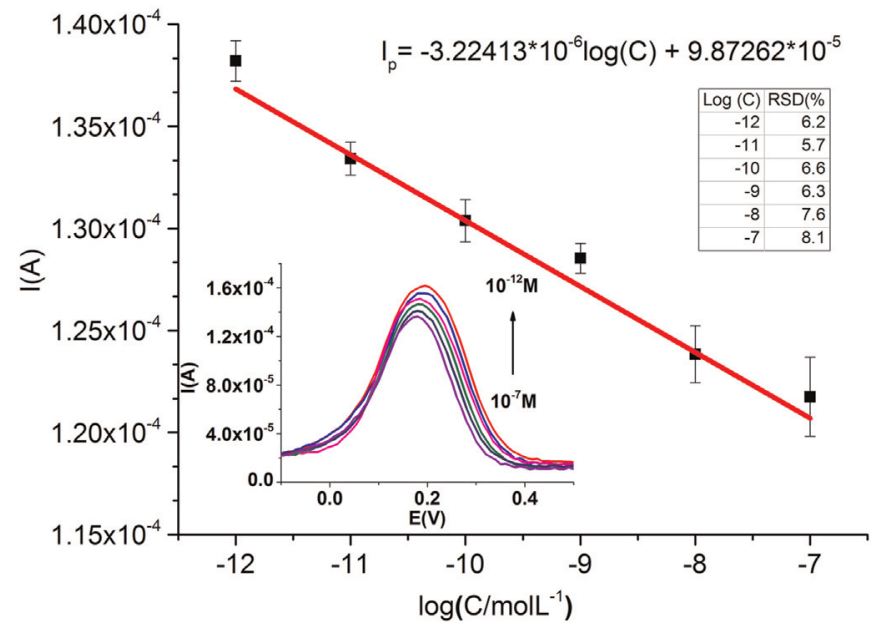

Fig. 6. Dependence of peak currents $\left(I_{\mathrm{p}}\right)$ of DPV spectra on the logarithmic concentration of target $\mathrm{cDNA} \log \left(\mathrm{C} / \mathrm{molL}^{-1}\right)$. Insets: DPV spectra recorded after hybridization with different concentration of target cDNA (varying from $10^{-7} \mathrm{M}$ to $10^{-12} \mathrm{M}$ with the corrected baselines) and the RSD (\%) value of all devices measured.

before (Lu et al., 2009; Lei et al., 2011). The interaction between $\pi$ electrons is still strong enough to maintain most of dsDNA molecules close enough to the surface of rGO, which leads to a more negatively charged electrode surface and further decreased redox currents. In this work, the interaction between target cDNA and rGO has been minimized by saturating the rGO surface with the highly concentrated probe ssDNA (at least 10 times more concentrated than target cDNA as mentioned above), and thus, the target cDNA mainly bonded to the probe ssDNA instead of bonding to rGO via $\pi-\pi$ bonds.

DPV measurements have been carried out to evaluate the sensitivity of this DNA biosensor in the ferricyanide system by varying the concentration of target cDNA from $10^{-7} \mathrm{M}$ to $10^{-12} \mathrm{M}$. As shown in Fig. 6, the peak currents taken from the DPV spectra increase linearly with the decrease of target cDNA concentration ranging from $10^{-7} \mathrm{M}$ to $10^{-12} \mathrm{M}$. The fitting equation is:

$I_{p}=-3.22413 \times 10^{-6} \log (C)+9.87262 \times 10^{-5}$

where $C$ stands for the concentration of target cDNA. The $R^{2}$ value for this equation is 0.9875 . According to $3 \sigma$ (the standard deviation of noise, $n=7$ ) obtained from the blank reference samples, the limit of detection calculated from the best set of results is found to be $1.58 \times 10^{-13} \mathrm{M}$. The selectivity of this DNA sensor has also been evaluated by adding non-complementary target DNA onto the same ssDNA-rGO-graphene electrodes. The corresponding changes in the redox currents are $7.7 \%$ of that obtained from the complementary target DNA with the same concentration and under the same measurement conditions. The stability was investigated by immersing this DNA sensor in the PBS buffer and increases in the redox currents were observed after $3 \mathrm{~h}$, which may be caused by the degradation of ssDNA. A limited number of parallel ssDNArGO-graphene electrodes were used to investigate the reproducibility towards the detection of different target cDNA concentrations. The RSD values of all the devices measured are shown in the right inset of Fig. 6. The DNA sensor shows a wider linear dynamic range and a lower detection limit compared with those DNA sensors modified with polymer (Zhu et al., 2006), carbon nanotube (Zhang et al., 2009) or bare graphene quantum dot (Zhao et al., 2011), which are mainly attributed to the larger electroactive area and higher electron transport rate inherited from rGO-graphene structure. However, in comparison with those covalently immobilized graphene DNA sensors or those modified with metal nanoparticles, there is still room for improvement in sensor sensitivity
(Liu et al., 2013), reproducibility (Du et al., 2010) and stability (Zhu et al., 2012).

\section{Conclusions}

A novel rGO-graphene double-layer electrode has been developed and its application in label-free detection of DNA has been demonstrated. In comparison with electrodes of bare graphene, rGO or GO-graphene, an enhanced redox current on the rGOgraphene double-layer electrode was observed, which is attributed to a combination of the huge number of electroactive sites in reduced graphene oxide and the highly conductive nature of pristine graphene. The immobilization process developed in the work offers a rapid, convenient and low-cost technique for effective sensing of DNAs without the need to involve deleterious chemicals. The rGO-graphene based sensor exhibits a wide linear range and a good sensitivity for the label-free DNA detection, however the sensor reproducibility needs to be further improved.

\section{Acknowledgement}

We acknowledge the financial support from the UK EPSRC under grand no. EP/M006301/1 and the financial support from China Scholarship Council. We also acknowledge Dr Kamrul Islam for the helpful discussions of the manuscript.

\section{References}

Avent, N.D., Madgett, T.E., Maddocks, D.G., Soothill, P.W., 2009. Curr. Opin. Obstet. Gynecol. 21, 175-179.

Bard, A.J., Faulkner, L.R., 1980. Electrochemical Methods: Fundamentals and Applications. Wiley, New York.

Batchelor-McAuley, C., Wildgoose, G.G., Compton, R.G., 2009. Biosens. Bioelectron. 24, 3183-3190.

Bo, Y., Wang, W., Qi, J., Huang, S., 2011. Analyst 136, 1946-1951.

Cao, X., 2014. Microchim. Acta 181, 1133-1141.

Chang, H., Tang, L., Wang, Y., Jiang, J., Li, J., 2010. Anal. Chem. 82, 2341-2346.

Davies, T.J., Hyde, M.E., Compton, R.G., 2005. Angew. Chem. Int. Ed. 44, 5121-5126. Dong, X., Huang, W., Chen, P., 2010a. Nanoscale Res. Lett. 6, 1-6.

Dong, X., Shi, Y., Huang, W., Chen, P., Li, L.-J., 2010b. Adv. Mater. 22, 1649-1653.

Dreyer, D.R., Park, S., Bielawski, C.W., Ruoff, R.S., 2010. Chem. Soc. Rev. 39, 228-240. Du, M., Yang, T., Jiao, K., 2010. J. Mater. Chem. 20, 9253-9260.

Gao, W., Alemany, L.B., Ci, L., Ajayan, P.M., 2009. Nat. Chem. 1, 403-408.

Gavalas, V.G., Law, S.A., Christopher Ball, J., Andrews, R., Bachas, L.G., 2004. Anal. Biochem. 329, 247-252.

Gómez-Navarro, C., Weitz, R.T., Bittner, A.M., Scolari, M., Mews, A., Burghard, M., Kern, K., 2007. Nano Lett. 7, 3499-3503.

He, S., Song, B., Li, D., Zhu, C., Qi, W., Wen, Y., Wang, L., Song, S., Fang, H., Fan, C., 2010. Adv. Funct. Mater. 20, 453-459.

Houdebine, L.-M., 2007. Transgenic animal models in biomedical research. In: Sioud, M. (Ed.), Target Discovery and Validation Reviews and Protocols. Humana Press, Norway, pp. 163-202.

Hu, Y., Li, F., Bai, X., Li, D., Hua, S., Wang, K., Niu, L., 2011. Chem. Commun. 47, $1743-1745$.

Kim, H.J., Lee, S.-M., Oh, Y.-S., Yang, Y.-H., Lim, Y.S., Yoon, D.H., Lee, C., Kim, J.-Y., Ruoff, R.S., 2014. Sci. Rep. 4, 5176.

Kotov, N.A., Dékány, I., Fendler, J.H., 1996. Adv. Mater. 8, 637-641.

Kovtyukhova, N.I., Ollivier, P.J., Martin, B.R., Mallouk, T.E., Chizhik, S.A., Buzaneva, E. V., Gorchinskiy, A.D., 1999. Chem. Mater. 11, 771-778.

Lei, H.Z., Mi, L.J., Zhou, X.J., Chen, J.J., Hu, J., Guo, S.W., Zhang, Y., 2011. Nanoscale 3, 3888-3892.

Lim, C.X., Hoh, H.Y., Ang, P.K., Loh, K.P., 2010. Anal. Chem. 82, 7387-7393.

Liu, A.-L., Zhong, G.-X., Chen, J.-Y., Weng, S.-H., Huang, H.-N., Chen, W., Lin, L.-Q., Lei, Y., Fu, F.-H., Sun, Z.-1, Lin, X.-H., Lin, J.-H., Yang, S.-Y., 2013. Anal. Chim. Acta 767 $50-58$.

Liu, F., Choi, J.Y., Seo, T.S., 2010. Biosens. Bioelectron. 25, 2361-2365.

Lu, C.-H., Yang, H.-H., Zhu, C.-L., Chen, X., Chen, G.-N., 2009. Angew. Chem.-Ger. Ed. 121, 4879-4881.

Lucchese, M.M., Stavale, F., Ferreira, E., Vilani, C., Moutinho, M., Capaz, R.B., Achete C., Jorio, A., 2010. Carbon 48, 1592-1597.

Niwa, O., Jia, J., Sato, Y., Kato, D., Kurita, R., Maruyama, K., Suzuki, K., Hirono, S. 2006. J. Am. Chem. Soc. 128, 7144-7145.

Novoselov, K.S., Falko, V.I., Colombo, L., Gellert, P.R., Schwab, M.G., Kim, K., 2012 
Nature 490, 192-200.

Novoselov, K.S., Geim, A.K., Morozov, S.V., Jiang, D., Zhang, Y., Dubonos, S.V., Grigorieva, I.V., Firsov, A.A., 2004. Science 306, 666-669.

Pandey, D., Reifenberger, R., Piner, R., 2008. Surf. Sci. 602, 1607-1613.

Patil, A.J., Vickery, J.L., Scott, T.B., Mann, S., 2009. Adv. Mater. 31, 3159-3164.

Ramesha, G.K., Sampath, S., 2009. J. Phys. Chem. C 113, 7985-7989.

Shin, H.J., Kim, K.K., Benayad, A., Yoon, S.M., Park, H.K., Jung, I.S., Jin, M.H., Jeong, H. K., Kim, J.M., Choi, J.Y., 2009. Adv. Funct. Mater. 19, 1987-1992.

Silva, M.M.S., Dias, A.C.M.S., Silva, B.V.M., Gomes-Filho, S.L.R., Kubota, L.T., Goulart, M.O.F., Dutra, R.F., 2014. J. Chem. Technol. Biotechnol. 90, 194-200.

Su, Q., Pang, S., Alijani, V., Li, C., Feng, X., Müllen, K., 2009. Adv. Mater. 21, 3191-3195.
Teixeira, S., Burwell, G., Castaing, A., Gonzalez, D., Conlan, R.S., Guy, O.J., 2014. Sens. Actuat. B-Chem 190, 723-729.

Wan, Y., Wang, Y., Wu, J., Zhang, D., 2010. Anal. Chem. 83, 648-653.

Wu, X., Chai, Y., Yuan, R., Su, H., Han, J., 2013. Analyst 138, 1060-1066.

Zhang, X., Jiao, K., Liu, S., Hu, Y., 2009. Anal. Chem. 81, 6006-6012.

Zhao, J., Chen, G., Zhu, L., Li, G., 2011. Electrochem. Commun. 13, 31-33.

Zhao, J., Pei, S., Ren, W., Gao, L., Cheng, H.-M., 2010. ACS Nano 4, 5245-5252.

Zhu, L., Luo, L., Wang, Z., 2012. Biosens. Bioelectron. 35, 507-511.

Zhu, N., Chang, Z., He, P., Fang, Y., 2006. Electrochim. Acta 51, 3758-3762. 